\title{
Viewpoint
}

\section{Comment on Johnston (2000): Commentary on Burgin and Wild (1967): Stock control-experience and usable theory. J Opl Res Soc 51: 1111}

I was interested to see the commentary ${ }^{1}$ on the republication of Burgin and Wild's 1967 paper $^{2}$ in the October 2000 $J O R S$, having been much involved in stock control in the 1960s. Trained by BOR Ltd, I implemented their rules at Mather \& Platt Ltd, and later applied similar techniques at BOAC.

In retrospect, I think that the mathematics, though exciting, was the easy bit. Once one had linked the service level not just to the probability of running out during a lead time but also to the frequency of re-ordering, ie how often one would run the risk, sensible levels could easily be computed. Which probability distribution one used seemed not to matter much.

There were, and still are, more serious challenges. If forecasting by extrapolation of past demand, how do you know whether a large withdrawal indicates more usage in future, or whether for example some other OR person has recommended replacing all the light bulbs in the factory at one go rather than individually as they fail? I agree about the need to influence lead times. When we turned our attention to repairable components, we realised that most of the observed repair times was spent queueing: if you run the workshops very short of some items, they have to repair them PDQ.

The greatest difficulty is in determining the cost of a stock-out. Setting this equal for all items, so that the overall service level is maximised for a given investment, leads to very little stock of expensive items but a lifetime's supply of cheap ones. Although highly economic, this fails to take into account that it is difficult to substitute for costly items, whereas one can always make short screws out of long ones, for instance, or use stainless steel instead of mild. I found that setting the stock-out cost proportional to the square root of the item value gave robust results, but then I am fond of square-root relationships.

I ought also to mention the very tricky matter of implementation. If you suddenly bring in a new set of reorder levels, however they have been calculated, many items will be found to have stock below reorder point, so that a tidal wave of orders is generated, putting short-term pressure on both order processing and funding. Meanwhile, the running down of stocks of obsolete and slow-moving items will continue no faster than before. Unless there is extreme caution, the promised savings can be hard to demonstrate.

London, UK

JN Floyer

\section{References}

1 Johnston FR (2000). Commentary on Burgin and Wild (1967): Stock control-experience and usable theory. J Opl Res Soc 51: 1111.

2 Burgin TA and Wild AR (1967). Stock control-experience and usable theory. Opl Res $Q$ 18: 35-52. 